\title{
Predictive Ligament Balancing in Robotic TKA - One Year Clinical Outcomes from a Multicenter Study
}

\author{
John M. Keggi, MD ${ }^{1}$, Jeffrey M. Lawrence, $\mathrm{MD}^{2}$, Amber L. Randall, $\mathrm{MD}^{3}$, \\ Jeffrey H. DeClaire ${ }^{4}$, Corey E. Ponder ${ }^{5}$, Jan Koenig ${ }^{6}$, Sami Shalhoub MS ${ }^{7}$, \\ Edgar Wakelin ${ }^{7}$, Christopher Plaskos $\mathrm{PhD}^{7}$ \\ ${ }^{1}$ Orthopaedics New England, Middlebury, CT. \\ ${ }^{2}$ Gundersen Health System, Viroqua, WI. \\ ${ }^{3}$ Flagstaff Bone and Joint, Flagstaff, AZ. \\ ${ }^{4}$ DeClaire LaMacchia Orthopaedic Institute, Rochester Hills, MI. \\ ${ }^{5}$ Oklahoma Sports and Orthopedics Institute, Edmond, OK, USA. \\ ${ }^{6}$ NYU Winthrop University Hospital, Long Island, NY, USA \\ ${ }^{7}$ Corin USA. Raynham, MA. \\ john.keggi@gmail.com, christopher.plaskos@coringroup.com
}

\begin{abstract}
This study reports on the one-year clinical results and patient reported outcomes (PROMs) associated with a new 'predictive balance' tibia-cut first total knee arthroplasty (TKA) technique that uses a robotic ligament tensioner. PROMs are compared to registry data and historical results in the literature.

Five hundred and thirty-three patients were prospectively enrolled and underwent robotic TKA (mean age: 67.7 \pm 8.4 ; females: 320; BMI: 31.2 \pm 4.9 ). Pre-op, three, six and 12-month WOMAC, UCLA activity scale, and HSS-Patient satisfaction assessments were completed by 533, 352, 314, and 256 patients, respectively, and compared to WOMAC registry data from the Shared Ortech Aggregated Repository (SOAR) and to historical satisfaction reports in the literature.

Despite having equivalent baseline PROM scores, predictive balance patients had significantly higher WOMAC scores at all post-operative timepoints $(\mathrm{p}<0.001)$ and higher UCLA activity scale scores at $3 \mathrm{M}$ and $6 \mathrm{M}(\mathrm{p}<0.013)$. Overall patient satisfaction in the predictive balance cohort was $91.2 \%, 92.4 \%$, and $96.5 \%$ at $3 \mathrm{M}, 6 \mathrm{M}$ and $1 \mathrm{YR}$, respectively. Average length of stay was 1.6 days $( \pm 0.8)$. Surgical complications in this cohort were typical of TKA.

Limitations to this study include the lack of a closely matched control group. Nonetheless, first year results are promising with improved objective measures compared to large registry databases and recently reported patient satisfaction measures.
\end{abstract}


Predictive Ligament Balancing in Robotic TKA - One Year Clinical Outcomes from a ... Keggi ett al.

\section{Introduction}

Total knee arthroplasty (TKA) soft tissue balancing methods have evolved from subjective surgeon feel to manual tensiometers, load sensing inserts [1] and computer navigated gap balancing techniques [2]. These allow the surgeon to assess the soft-tissue envelope after all bone cuts are made and can guide subsequent releases. More recently, a robotic ligament tensioning device has been deployed which characterizes the soft tissue envelope through the continuous range of motion after the tibial cut only [3,4]. It then provides a predictive model of the final compartment gaps through the entire range of motion based on the proposed femoral implant plan which can then be adjusted and re-assessed prospectively to optimize the final outcome. Initial results on the accuracy and early three to six-month outcomes of this technique have been reported [4,5]; however longer-term data is lacking.

This study reports on first year clinical results and patient reported outcomes (PROMs) of this novel technique, including knee pain, stiffness, and function, and patient satisfaction, which are compared to registry data and historical results in the literature.

\section{Methods}

\subsection{Surgical Technique}

The full surgical technique has been previously described using the Omni Apex knee, OmniBotics navigation system and BalanceBot automated distraction device (Corin USA, Raynham MA) [3,5]. The BalanceBot is inserted into the knee after the tibial cut in a tibia-first technique and before any ligament releases (figure 1a). The device applies a known, computer-controlled distraction force while the knee is taken through a range of motion. The gaps are characterized in the medial and lateral compartments from full extension through more than 90 degrees flexion. Femoral cut planning is then carried out with an instant display of the predicted gaps and laxity profile (Predictive Balance ${ }^{\mathrm{TM}}$ ) based on virtual manipulation of the femoral component in all planes (figure 1b). After femoral cuts, results are confirmed when the femoral trial is in place using the BalanceBot as a virtual tibial trial that assesses the laxity profile, compartment pressures and knee kinematics through the continuous range of motion.

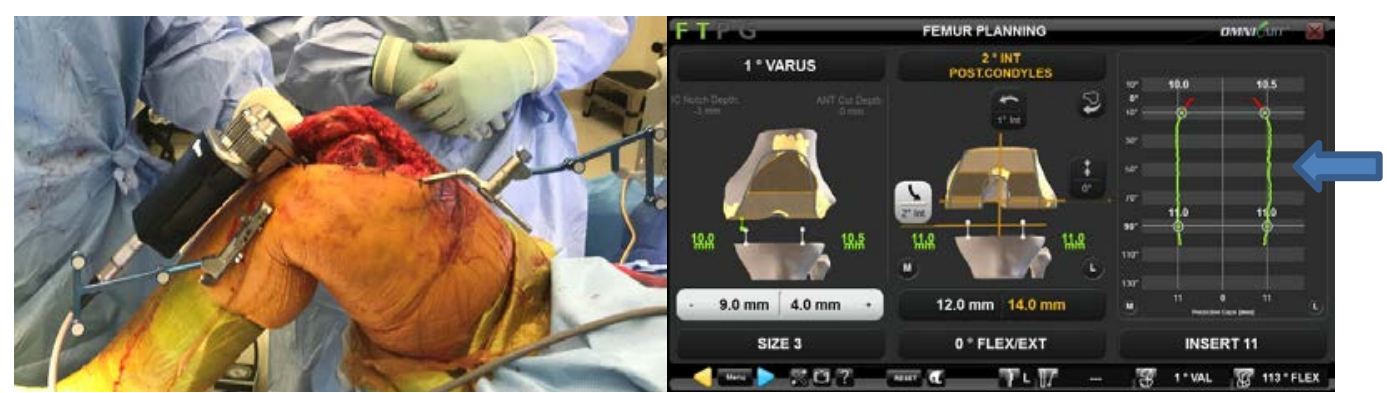

Figure 1 a) The BalanceBot is inserted into the knee after the tibial resection but before femoral resections. b) femoral planning is performed with predictive gap curves (blue arrow) to achieve a balanced knee throughout the range of motion.

\subsection{Study Methods and Patients}

This is an ongoing prospective multicenter study evaluating patient reported outcomes following cruciate sacrificing TKA with a PS or ultra-congruent tibial insert with the BalanceBot system. 
Predictive Ligament Balancing in Robotic TKA - One Year Clinical Outcomes from a ... Keggi ett al.

KOOS/WOMAC, UCLA, HSS-Patient Satisfaction, and PROMIS-10 scores are collected preoperatively and post-operatively at three months (3M), six months (6M) and one year (YR). Between November 2017 and January 2020, 533 patients were enrolled and underwent robotic TKA using this surgical technique (mean age: $66.2 \pm 8.1$; females: 320 ; BMI: $31.3 \pm 5.3$ ). Three-month, $6 \mathrm{M}$ and $1 \mathrm{YR}$ assessments were completed by 352, 314, and 256 patients, respectively, and compared to registry data from the Shared Ortech Aggregated Repository (SOAR) and to historical reports in the literature. SOAR is a TJA PROM repository run by Ortech [3], an independent clinical data collection entity, and it includes data from over five thousand TKAs from a diverse cross-section of participating hospitals, teaching institutions and clinics across the United States and Canada who collect outcomes data. Preand post-operative PROM data were compared between the Predictive Balance and SOAR groups using a two-tailed t-test for non-equal variance. Normality was assessed using Shapiro-Wilk test.

\section{Results}

When comparing the baseline PROM scores between the Predictive Balance and SOAR groups, robotic patients had equivalent WOMAC scores $(\mathrm{p}>0.07)$ and UCLA activity scale $(\mathrm{p}=0.545)$, figure 2a. While all scores improved over time, statistically higher scores were observed in the robotic group for all categories at all post-operative timepoints for the WOMAC scale ( $<<0.001)$, and for the UCLA score at $3 \mathrm{M}$ ( 5.0 vs $5.3, \mathrm{p}=0.00026)$ and $6 \mathrm{M}$ ( 5.2 vs $5.7, \mathrm{p}<0.0001)$. Overall patient satisfaction in the Predictive Balance cohort was 91.2\%, 92.4\% and 96.5\% at 3M, 6M and 1YR (figure 2b). The average length of hospital stay was 1.6 days $( \pm 0.8)$. Surgical complications in this cohort included:

1. Manipulation Under Anesthesia for stiffness x 7

2. Pulmonary Embolism $\mathrm{x} 1$

3. Effusion $\mathrm{x} 2$ (treated with aspiration)

4. Hemarthrosis treated with arthrocentesis $\mathrm{x} 1$

5. Wound dehisence from fall $\mathrm{x} 1$

7. Deep vein thrombosis $\mathrm{x} 2$

8. Infection (four months post-op) $\mathrm{x} 1$

\section{Discussion}

Consideration of soft tissue balance has been demonstrated to correlate with patient reported outcomes when using a pressure-sensing device [1,7]. However, the data is acquired only at certain poses and not on a continuous basis. Additionally, specific gap data is not acquired under known or reproducible load conditions with any of the previously available systems. Acquisition of continuous gap data on the other hand has been shown to be very reproducible [5]. We postulated that the ability to use gap data under known loading conditions through the continuous range of motion prospectively would allow femoral cut planning that resulted in optimum balance and better long-term result. While the study group patients had equivalent baseline knee pain, stiffness and function than registry patients, study patients showed significantly better improvements in all areas up to one year post-operatively compared to registry data. UCLA activity scores were equal between the two groups at baseline and significantly improved at three months and six months. These gains may reflect better ligament balance in the study group. Gustke reported a trend toward improved WOMAC scores in "balanced" knees using a pressure sensing device [1]. A subsequent study by Gustke reported the routine use of two or three (and up to as many as 8) releases in order to achieve balance using the same device [8]. By contrast the device used in our study allows placement of the femoral component to be made 
Predictive Ligament Balancing in Robotic TKA - One Year Clinical Outcomes from a ... Keggi ett al.
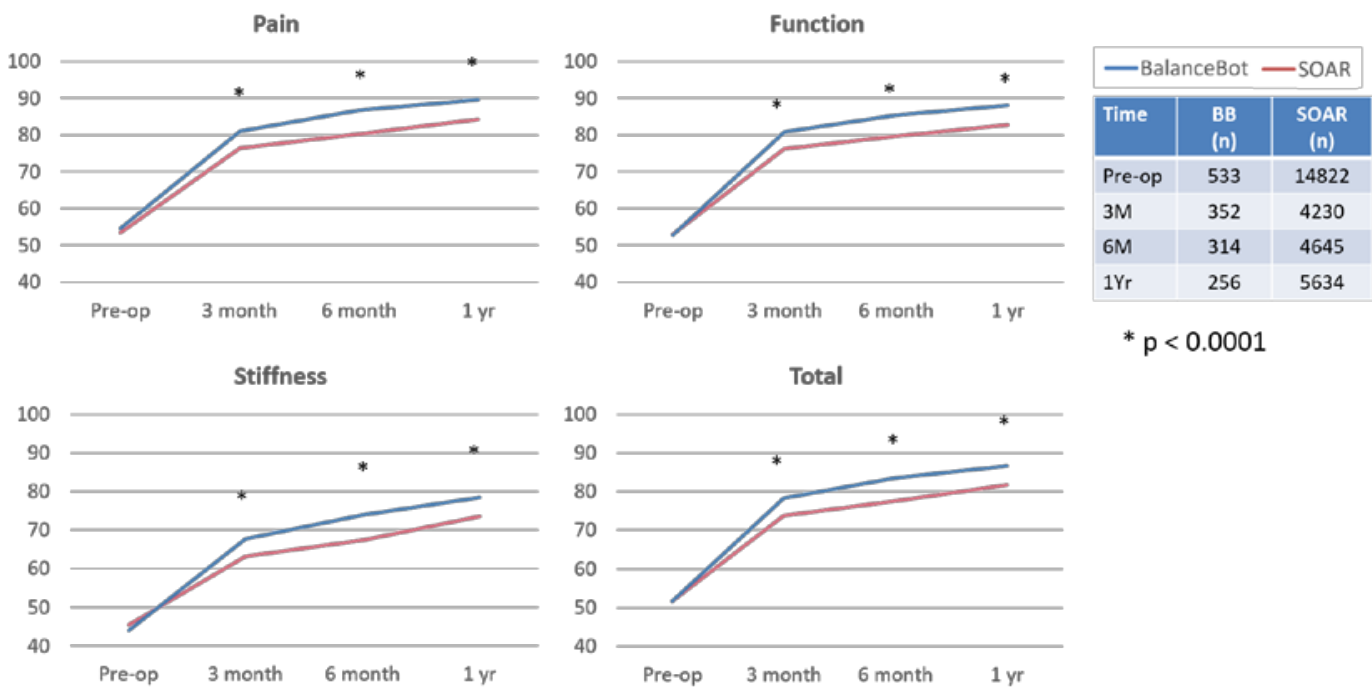

Figure 2a: Improvements in knee stiffness, function, pain and total WOMAC scores were seen in the Predictive Balance group over the SOAR group at 3M, 6M, and 1 Yr post-operatively $(\mathrm{p}<0.001)$, despite having equivalent baseline scores.

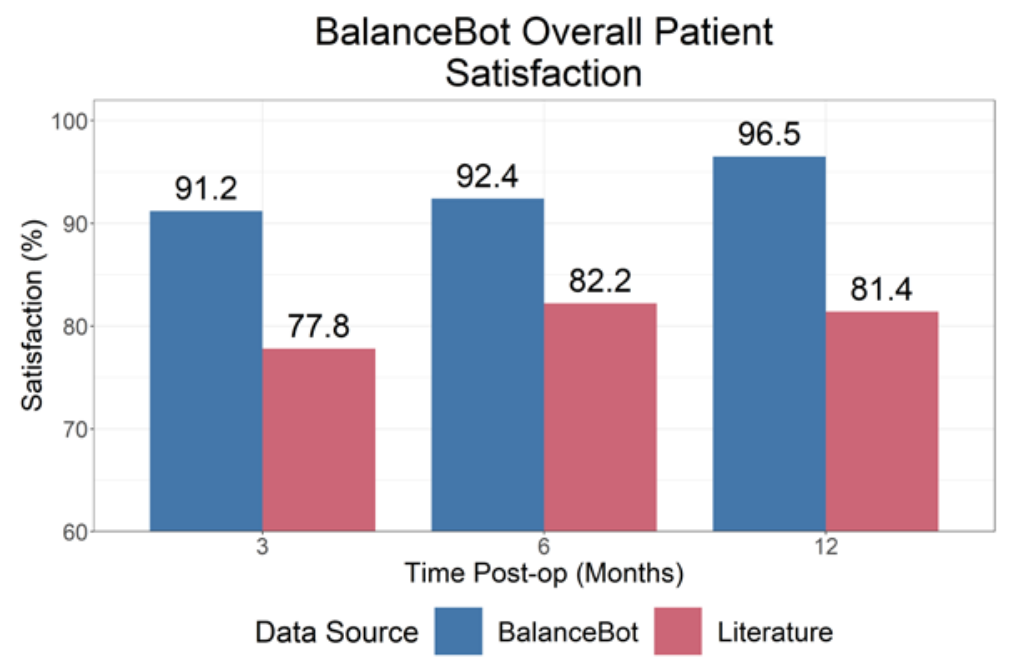

Figure 2b: Overall patient satisfaction in the Predictive Balance cohort was 91.2\%, 92.4\% and $96.5 \%$ at $3 \mathrm{M}, 6 \mathrm{M}$ and $12 \mathrm{M}$, which compares favorably to satisfaction data commonly reported in the literature*. [Mean patient satisfaction values were computed from the following literature sources using weighted averages: 1. Turcot, JOA 2013. 2. Van Onsem JOA 2016. 3. Vissers BMCMD 2010. 4. Kiran, JOA 2015. 5. Bourne, CORR 2010. 6. Heck, CORR 1998. 7. Baker, JBJS, 2007. 8. Noble, CORR 2006. 9. Robertsson, Acta 2000. 10. Lange, JOA 2018. 11. AOANJRR PROMs Pilot Project Final Report, Feb 2020] 
Predictive Ligament Balancing in Robotic TKA - One Year Clinical Outcomes from a ... Keggi ett al.

prospectively with the goal of reducing subsequent ligament releases which may improve patient function and comfort as evidenced by the low rate of manipulations out to 1 year $(2.7 \%$ vs $6.8 \%, p=$ 0.011 ) [9]. This may be related to the higher rates of satisfaction reported in the study patients compared to the historical literature [10-13] (Figure 2b).

Limitations to this study include the lack of a closely matched control group. Nonetheless, the firstyear results are promising with improved objective measures and subjective outcomes compared to a large registry database and recently reported patient satisfaction measures. Assessment of the soft tissue envelope through the continuous range of motion using gap data may provide better patient outcomes.

\section{References}

1. Gustke KA, Golladay GJ, Roche MW, Elson LC, Anderson CR. A New Method for Defining Balance: Promising Short-Term Clinical Outcomes of Sensor-Guided TKA. J Arthroplasty. 2014 May 1;29(5):955-60.

2. Joseph J, Simpson PM, Whitehouse SL, English HW, Donnelly WJ. The use of navigation to achieve soft tissue balance in total knee arthroplasty - a randomised clinical study. Knee. 2013 Dec;20(6):401-6.

3. Shalhoub S, Moschetti WE, Dabuzhsky L, Jevsevar DS, Keggi JM, Plaskos C. Laxity Profiles in the Native and Replaced Knee-Application to Robotic-Assisted Gap-Balancing Total Knee Arthroplasty. J Arthroplasty. 2018;33(9):3043-8.

4. Keggi JM, Lawrence JM, Randall A, Declaire JH, Shalhoub S, Plaskos C. Early Clinical Outcomes of a Novel Predictive Ligament Balancing Technique for Total Knee Arthroplasty. CAOS Int'l. New York, June 2019

5. Shalhoub S, Lawrence JM, Keggi JM, Randall AL, DeClaire JH, Plaskos C. Imageless, roboticassisted TKA combined with a robotic tensioning system can help predict and achieve accurate postoperative ligament balance. Arthroplasty Today. 5 (2019) 334-340.

6. https://ortechsystems.com/ accessed on Jan 31, 2020.

7. Meneghini RM, Ziemba-Davis MM, Lovro LR, Ireland PH, Damer BM. Can Intraoperative Sensors Determine the "Target" Ligament Balance? Early Outcomes in Total Knee Arthroplasty. J Arthroplasty. 2016 Oct 1;31(10):2181-7.

8. Gustke KA, Golladay GJ, Roche MW, Elson LC, Anderson CR. A Targeted Approach to Ligament Balancing Using Kinetic Sensors. J Arthroplasty. 2017 Jul 1;32(7):2127-32.

9. Issa, K., B.H. Kapadia, M. Kester, H.S. Khanuja, R.E. Delanois, and M.A. Mont. Clinical, objective, and functional outcomes of manipulation under anesthesia to treat knee stiffness following total knee arthroplasty. The Journal of arthroplasty, 2014; 29(3): 548-552.

10. Lange JK, Lee Y-Y, Spiro SK, Haas SB. Satisfaction Rates and Quality of Life Changes Following Total Knee Arthroplasty in Age-Differentiated Cohorts. J Arthroplasty. 2018 May 1;33(5):1373-8.

11. Turcot K, Sagawa Y Jr, Fritschy D, Hoffmeyer P, Suvà D, Armand S. How Gait and Clinical Outcomes Contribute to Patients' Satisfaction Three Months Following A Total Knee Arthroplasty. J Arthroplasty. 2013 Sep 1;28(8):1297-300.

12. Van Onsem S, Van Der Straeten C, Arnout N, Deprez P, Van Damme G, Victor J. A New Prediction Model for Patient Satisfaction After Total Knee Arthroplasty. J Arthroplasty. 2016 Dec 1;31(12):2660-2667.e1.

13. Kiran A, Bottomley N, Biant LC, Javaid MK, Carr AJ, Cooper C, et al. Variations In Good Patient Reported Outcomes After Total Knee Arthroplasty. J Arthroplasty. 2015 Aug 1;30(8):1364-71. 